\title{
Knockdown of circ_0011946 targets miR- 216a-5p/BCL2L2 axis to regulate proliferation, migration, invasion and apoptosis of oral squamous cell carcinoma cells
}

Ying Zhou', Shuhong Zhang ${ }^{1 *}$, Zhonghan Min², Zhongwei Yu', Huaiwei Zhang ${ }^{1}$ and Jiao Jiao'

\begin{abstract}
Background: Circular RNAs (circRNAs) are implicated in the development of oral squamous cell carcinoma (OSCC). The aim of current research is to elucidate the role and mechanism of circ_0011946 in the functional behaviors of OSCC cells.

Methods: Circ_0011946, microRNA (miR)-216a-5p, B cell lymphoma-2-like 2 protein (BCL2L2) abundances were exposed by quantitative reverse transcription polymerase chain reaction (qRT-PCR) or western blot. Cell proliferation, migration, invasion and apoptosis were detected by MTT, colony formation assay, transwell, woundhealing and flow cytometry assays, respectively. Target correlation was tested by dual-luciferase reporter and RNA pull-down assays. An in vivo xenograft experiment was employed to investigate the function of circ_0011946 on tumor growth in vivo.

Results: Circ_0011946 and BCL2L2 levels were increased, while miR-216a-5p level was decreased in OSCC tissues and cells. Circ_0011946 knockdown impeded proliferation, migration, and invasion, but promoted apoptosis in OSCC cells. Circ_0011946 functioned as a sponge for miR-216a-5p, and BCL2L2 was targeted by miR-216a-5p. Besides, miR-216a-5p or BCL2L2 knockdown partly attenuated the inhibitory influences of circ_0011946 silence or miR-216a-5p overexpression on OSCC cell progression. Furthermore, circ_0011946 post-transcriptionally regulated BCL2L2 through sponging miR-216a-5p. Moreover, circ_0011946 knockdown constrained OSCC tumor growth in vivo.
\end{abstract}

Conclusion: Circ_0011946 silence repressed OSCC cell proliferation, migration, and invasion, but promoted apoptosis through the regulation of the miR-216a-5p/BCL2L2 axis.

Keywords: Oral squamous cell carcinoma, circ_0011946, miR-216a-5p, BCL2L2

\footnotetext{
* Correspondence: xiaolu262119@163.com

'Department of Dentistry, Jiaxing Hospital of Traditional Chinese Medicine, Jiaxing University, No. 1501 Zhongshan East Road, Jiaxing, Zhejiang 314001, China

Full list of author information is available at the end of the article
}

(c) The Author(s). 2021 Open Access This article is licensed under a Creative Commons Attribution 4.0 International License, which permits use, sharing, adaptation, distribution and reproduction in any medium or format, as long as you give appropriate credit to the original author(s) and the source, provide a link to the Creative Commons licence, and indicate if changes were made. The images or other third party material in this article are included in the article's Creative Commons licence, unless indicated otherwise in a credit line to the material. If material is not included in the article's Creative Commons licence and your intended use is not permitted by statutory regulation or exceeds the permitted use, you will need to obtain permission directly from the copyright holder. To view a copy of this licence, visit http://creativecommons.org/licenses/by/4.0/ The Creative Commons Public Domain Dedication waiver (http://creativecommons.org/publicdomain/zero/1.0/) applies to the data made available in this article, unless otherwise stated in a credit line to the data. 


\section{Background}

Oral cancer is a common oral disease, and the major risk factors are tobacco use and alcohol consumption [1]. Oral squamous cell carcinoma (OSCC) is one of the primary cases of oral cancer, with low survival rate and high incidence [2]. Exploring the pathogenesis of OSCC may support a new therapeutic strategy for OSCC.

Noncoding RNAs are related to OSCC progression [3]. Circular RNAs (circRNAs) with covalently closed structure [4], which have been implicated in the development of various cancers, such as OSCC [5, 6]. For instance, circ_0000140 can inhibit cell proliferation, metastasis by mediating miR-31/Hippo pathway in OSCC [7]. Circ 002178 can promote cell proliferation and migration by activating the protein kinase $\mathrm{B} /$ mammalian target of rapamycin (AKT/mTOR) pathway in OSCC [8]. CircRNA hsa_circ_0011946 (circ_0011946), derived from the back-splicing of exons of Scm polycomb group protein homolog 1 (SCMH1), is capacity of promoting OSCC cell development by regulating proliferating cells nuclear antigen (PCNA) [9]. Nevertheless, our understanding of the mechanisms of circ_0011946 regulation in OSCC is still limited.

MicroRNAs (miRNAs) are another type of small noncoding RNA molecules, and dysregulated miRNAs are associated with OSCC progression [10,11]. A previous study uncovered that miR-216a-5p can repress the malignant phenotypes of OSCC via regulating eukaryotic translation initiation factor $4 \mathrm{~B}$ (EIF4B) [12]. B cell lymphoma-2-like 2 protein (BCL2L2) belongs to the BCL2 family that exerts an important role in human cancers [13]. Moreover, BCL2L2 constrained the tumorigenesis of human cancers, like hepatocellular carcinoma and non-small cell lung cancer $[14,15]$. More importantly, BCL2L2 can promote tumor development by increasing cell proliferation in OSCC [16]. However, whether miR-216a-5p and BCL2L2 participate in the regulation of circ_0011946 in OSCC development is undiscovered.

In present research, we scrutinized the influences and mechanism of circ_0011946 in the malignant phenotypes of OSCC cells.

\section{Methods}

\section{Tissues collection}

OSCC tumor tissues and paired adjacent normal samples ( $>2 \mathrm{~cm}$ from tumor tissue) were collected 30 OSCC patients, who undergo surgery between 2012 and 2015 at Jiaxing Hospital of Traditional Chinese Medicine, Jiaxing University. Patients received any other therapy before surgery were excluded. All tissues were stored in liquid nitrogen. The clinical-parameters of OSCC patients were provided in Table 1 . The research got the permission of the ethics committee of Jiaxing Hospital
Table 1 The clinicopathological parameters of patients with OSCC $(n=30)$

\begin{tabular}{llll}
\hline Parameter & Case & \multicolumn{2}{l}{ Circ_0011946 expression } \\
\cline { 3 - 4 } & & Low $(\boldsymbol{n}=14)$ & High $(\boldsymbol{n}=\mathbf{1 6})$ \\
\hline Age (years) & 15 & 6 & 9 \\
$\leq 60$ & 15 & 8 & 7 \\
$>60$ & & & 6 \\
Sex & 13 & 7 & 10 \\
Female & 17 & 7 & \\
Male & 16 & 11 & 5 \\
Tumor size & 14 & 3 & 11 \\
$\leq 5 \mathrm{~cm}$ & & & 5 \\
$>5 \mathrm{~cm}$ & 14 & 9 & 11 \\
TNM stages & 16 & 5 & \\
I-II & & & \\
III-IV & & &
\end{tabular}

of Traditional Chinese Medicine, Jiaxing University with the obtained written informed consent.

\section{Cell culture and transfection}

OSCC cell lines (CAL27 and SCC25) were procured from Procell (Wuhan, China) and the culture medium is comprised of Dulbecco Modified Eagle's medium (DMEM; Gibco, Gran Island, NY, USA) and 10\% fetal bovine serum (Gibco). Human oral keratinocytes (HOK) were provided by Sciencell (Carlsbad, CA, USA) and cultured in oral keratinocyte medium (Sciencell) plus $1 \%$ oral keratinocyte growth supplement (Sciencell) and 1\% antibiotic solution (Sciencell). All cells were cultivated at $37^{\circ} \mathrm{C}$ in an incubator with $5 \% \mathrm{CO}_{2}$.

BCL2L2 overexpression vector (pc-BCL2L2) was constructed by inserting human BCL2L2 coding sequence (lacking the 3'UTR) into pcDNA3.1 vector (HonorGene, Changsha, China), and empty vector was regarded as negative control (pc-NC). Small interfering RNA (si) of circ_0011946, miR-216a-5p mimic, miR-216a-5p inhibitor, and their negative controls (si-NC, miRNA NC and inhibitor NC) were procured from GenePharma (Shanghai, China), and mentioned in Table 2 CAL27 and SCC25 cells $\left(1 \times 10^{5}\right)$ were introduced with $200 \mathrm{ng}$ of vectors or/and $30 \mathrm{nM}$ of miRNA mimic or/and $30 \mathrm{nM}$ of miRNA inhibitor or/and $50 \mathrm{nM}$ of siRNA using Liposomal Transfection reagent (Yeasen, Shanghai, China). Transfection efficacy was analyzed after $24 \mathrm{~h}$.

\section{Quantitative reverse transcription PCR (qRT-PCR)}

RNA was lysed using Trizol (Applygen, Beijing, China). Nuclear and cytoplasmic RNA were prepared via the Cytoplasmic \& Nuclear RNA Purification Kit based on the manufacturer's suggestion (Norgen Biotek, Thorold, 
Table 2 Sequences of oligonucleotides and primers used in this research

\begin{tabular}{|c|c|c|}
\hline Name & Sequence & s $\left(5^{\prime}-3^{\prime}\right)$ \\
\hline si-circ_0011946\#1 & & 5'-AGCACUAGAUGCUUUGGUGCC-3' \\
\hline si-circ_001 1946\#2 & & 5'-UUGCUCUAGAUGCUUUGGUGC-3' \\
\hline si-circ_001 1946\#3 & & 5'-CUAGAUGCUUUGGUGCCAGGA-3' \\
\hline si-NC & & 5'-AACAGTCGCGTTTGCGACTGG-3' \\
\hline miR-216a-5p mimic & & 5'-UAAUCUCAGCUGGCAACUGUGA-3' \\
\hline miRNA NC & & 5'-UUCUCCGAACGUGUCACGU-3' \\
\hline miR-216a-5p inhibitor & & 5'-UCACAGUUGCCAGCUGAGAUUA-3' \\
\hline inhibitor NC & & $5^{\prime}-C \cup A A C G C A \cup G C A C A G \cup C G \cup A C G-3^{\prime}$ \\
\hline circ_0011946 & sense & 5'-TTCGACTCCCGAGACATCTT-3' \\
\hline & antisense & 5'-TCCTCTGTAGTGGAGCAGCA-3' \\
\hline $\mathrm{SCMH1}$ & sense & 5'-TCGTGGTGCAAACCTCTACC-3' \\
\hline & antisense & 5'-ACAGGGATCCTTCTCCTCCC-3' \\
\hline BCL2L2 & sense & 5'-GGCAAGAACTAGGGGCAGTT-3' \\
\hline & antisense & 5'-ATGCACAAGGAAGGGGGATG-3' \\
\hline miR-216a-5p & sense & 5'-GCCGAGGTAATCTCAGCTGG-3' \\
\hline & antisense & 5'-CAGTGCGTGTCGTGGAGT-3' \\
\hline U6 & sense & 5'-CTCGCTTCGGCAGCACA-3' \\
\hline & antisense & 5'-AACGCTTCACGAATTTGCGT-3' \\
\hline GAPDH & sense & 5'-GACAGTCAGCCGCATCTTCT-3' \\
\hline & antisense & 5'-GCGCCCAATACGACCAAATC-3' \\
\hline
\end{tabular}

ON, Canada). $1 \mu \mathrm{g}$ RNA was exposed to reverse transcription with specific Reverse Transcriptase kit (iGeneBio, Guangzhou, China). The synthesized cDNA with SYBR (Toyobo, Osaka, Japan) and primers (Table 3) (Genscript, Nanjing, China) was used for qRT-PCR assay. The primer sequences were displayed in Table 2 . U6 or GAPDH served as an internal control. Relative RNA level was decided by the $2^{-\Delta \Delta C t}$ method [17], with U6 or GAPDH as an interior control.

\section{Actinomycin D and RNase R treatment}

For the stability of circ_0011946, OSCC cells were stimulated with Actinomycin D $(2 \mu \mathrm{g} / \mathrm{mL}$; Cell Signaling Technology, Danvers, MA, USA) for $0,4,8,12$ or $24 \mathrm{~h}$. For RNase R treatment, RNA ( $1 \mu \mathrm{g})$ was incubated with $2 \mathrm{U}$ of RNase R (NovoBiotechnology, Beijing, China) at $37^{\circ} \mathrm{C}$ for $20 \mathrm{~min}$. Then, RNA was extracted, and circ 0011946, SCMH1 and GAPDH levels were uncovered by qRT-PCR.

\section{3-(4,5-dimethylthiazol-2-yl)-2,5-diphenyltetrazolium bromide (MTT)}

$1 \times 10^{4}$ cells/well CAL27 and SCC25 cells were inoculated onto the 96-well plates for $12 \mathrm{~h}$. Next, $10 \mu \mathrm{L}$ of MTT $(0.5 \mathrm{mg} / \mathrm{mL}$, Beyotime, Shanghai, China) was supplemented into per well. $4 \mathrm{~h}$ later, medium was replaced by $100 \mu \mathrm{L}$ of dimethyl sulfoxide (Thermo Fisher Scientific, Waltham, MA, USA). The absorbance was assessed using a microplate reader (Allsheng, Hangzhou, China) at $570 \mathrm{~nm}$.

\section{Colony formation assay}

Briefly, 200 cells were inoculated into the 6-well plates for 10 days, and were spotted with $0.1 \%$ crystal violet (Solarbio, Beijing, China). The images were photographed, and the number of colonies (>50 cells) was computed.

\section{Transwell and wound healing analyses}

Cell invasion was analyzed with Matrigel-coated transwell chambers (Costar, Corning, NY, USA). Cell migration was tested using transwell chambers without Matrigel and wound healing assay. For transwell assay, CAL27 and SCC25 cells $\left(1 \times 10^{4}\right.$ for migration assay, $3 \times$ $10^{4}$ for invasion analysis) in serum-free DMEM were plated into the superior chambers, whereas the lower chamber was supplemented with $500 \mu \mathrm{L}$ DMEM with $10 \%$ serum. $24 \mathrm{~h}$ upon incubation, migrated or invasive cells were spotted with $0.1 \%$ crystal violet, and examined under a microscope ( $\times 100$ magnification, Nikon, Tokyo, Japan) with at least 6 random fields.

For wound healing assay, $2 \times 10^{5}$ CAL27 and SCC25 cells after various transfections were placed into each well of 6-well plates until $80 \%$ of confluency was reached. Next, a "wound" was made using a $200 \mu \mathrm{L}$ pipette tip, and cells were allowed to migrate for additional $24 \mathrm{~h}$. The images ( $\times 100$ magnification) at 0 and $24 \mathrm{~h}$ were recorded. Relative migratory rate was calculated by the formula: (si-circ_0011946 (wound area)oh)-(si-circ_ $0011946_{\text {(wound }}$ area)24h $) /($ si-NC (wound area)0h $)-\left(\right.$ si-NC (wound $_{\text {woun }}$ area)24h).

\section{Flow cytometry}

Annexin V-FITC/Propidium Iodide (PI) apoptosis detection kit (Vazyme, Nanjing, China) was exploited for cell apoptosis analysis. A total of $2 \times 10^{5}$ of transfected CAL27 and SCC25 cells was spotted with Annexin VFITC and PI solution. Subsequently, cell apoptotic rate (Annexin $\mathrm{V}^{+} / \mathrm{PI}^{-}$and Annexin $\mathrm{V}^{+} / \mathrm{PI}^{+}$) were recognized by a flow cytometer (Jiyuan Biotech, Guangzhou, China).

\section{Western blot}

Protein was detached in RIPA buffer (Applygen) and quantitated by a protein quantitative kit (Beyotime). $20 \mu \mathrm{g} /$ lane Protein was segregated by sodium dodecyl sulfate-polyacrylamide gel and then transferred on polyvinylidene fluoride membrane (Solarbio). The membrane was blockaded in 5\% non-fat milk, and then nurtured with the antibody against BCL2L2 (ab190952, 1:2000, 
Abcam, Cambridge, MA, USA) overnight and a secondary antibody IgG labeled by horseradish peroxidase (HRP) (ab97200, 1:20,000, Abcam) for $2 \mathrm{~h}$. GAPDH (ab245356, 1:5000, Abcam) was used as a loading control. After hatching with ECL reagent (Amyjet Scientific, Wuhan, China), the blots were visualized by ChemiDoc XRS+ system (Bio-rad Laboratories, Richmond, California, USA) and analyzed Image J v1.8 software (NIH, Bethesda, MD, USA).

\section{Dual-luciferase reporter and RNA pull-down assays}

The miRNA-binding sites to circ_0011946 and human 3'UTRs was predicted by starBase database. The segment of circ_0011946 or BCL2L2 3'UTR harboring the predicted or mutant miR-216a-5p binding fragments were subcloned into the pmirGLO vector (Promega, Madison, WI, USA) to construct the luciferase report vector WTcirc_0011946, WT-BCL2L2-3'UTR, MUT-circ_0011946 or MUT-BCL2L2-3'UTR. These luciferase reporter vectors (200 ng) together with miR-216a-5p mimic or miRNA NC (30 nM) were transfected into CAL27 cells $\left(1 \times 10^{5}\right)$, respectively. $48 \mathrm{~h}$ upon incubation, the ratio of firefly to Renilla luciferase was estimated by the Dualluciferase reporter assay (Promega).

RNA pull-down assay was implemented through Magnetic RNA pull-down kit (Thermo Fisher Scientific). $1 \times$ $10^{7}$ CAL27 cells were lysed and hatched with the beadsconjugated biotin-labeled circ_0011946 probe or oligo probe (negative control) overnight at $4{ }^{\circ} \mathrm{C}$. RNA bound to beads was isolated, and circ_0011946 and miR-216a$5 \mathrm{p}$ abundances were discovered by qRT-PCR.

\section{Xenograft experiment}

$\mathrm{BALB} / \mathrm{c}$ nude mice (male, 5-week-old) were procured from Beijing Laboratory Animal Center (Beijing, China) and fed in specific pathogen-free microisolator cages. Lentiviruses expressing shRNA-circ_0011946 (sh-circ_ 0011946) and the negative control (sh-NC) were supplied by Ribobio (Guangzhou, China). Stable CAL27 cells were established by transducing with sh-circ 0011946 or sh-NC, and the selection of puromycin. Stable CAL27 cells $\left(3 \times 10^{6} /\right.$ mouse $)$ were hypodermically inoculated into the light flanks of the mice ( $n=6$ /group). Tumor volume was estimated every week based on the formula: length $\times$ width $^{2} / 2$. 4 weeks later, all mice were euthanized using $5 \%$ isoflurane. Tumor tissues were collected and weighed. circ_0011946, miR-216a-5p and BCL2L2 levels in tumor samples were measured. Proliferation of the tumors was assessed with paraffinembedded tumor tissues by immunohistochemistry under the standard method using the anti-Ki67 antibody (ab15580, 1:100 dilution, Abcam), biotinylated goat antirabbit IgG secondary antibody (ab64256, 1:300 dilution, Abcam) and a 3,3-diaminobenzidine (DAB) Kit (Vector
Laboratories, Peterborough, UK). The animal experiments got the approval of the Institutional Animal Care and Use Committee of Jiaxing Hospital of Traditional Chinese Medicine, Jiaxing University.

\section{Statistical analysis}

Data of three repetitions were represented as mean \pm standard deviation (SD) through GraphPad Prism 7 (GraphPad Inc., La Jolla, CA, USA). Student $t$-test or ANOVA followed by Tukey test was exploited to analyze the difference. The Pearson's rank correlation coefficient was exploited to ascertain the correlation of genes in OSCC tissues. $P<0.05$ was deemed as statistical significant.

\section{Results}

Circ_0011946 abundance is elevated in OSCC

To analyze whether circ_0011946 was associated with OSCC development, circ_0011946 expression was detected in OSCC tissues and cells. Circ_0011946 level was higher in OSCC tissues compared with normal specimens (Fig. 1A). Moreover, circ_0011946 expression was apparently upraised in CAL27 and SCC25 cells in comparison to HOK cells (Fig. 1B). Furthermore, the ringlike structure of circ_0011946 was certified, since circ 0011946 was resistant to Actinomycin D and RNase R digestion than linear GAPDH and linear SCMH1 (Fig. $1 C$ and D). Additionally, subcellular localization assays showed that circ_0011946 predominantly localized to the cytoplasm (Fig. 1E). Thus, circ_0011946 might contribute to OSCC development.

\section{Circ_0011946 knockdown constrains the tumorigenic phenotypes of OSCC cells}

To scrutinize the function of circ_0011946 in OSCC cell progression, siRNAs against circ_0011946 were introduced into CAL27 and SCC25 cells. Transfection of sicirc_0011946\#1 and \#2 effectively decreased circ_ 0011946 level, but si-circ_0011946\#3 did not affect the abundance (Fig. 2A). Meanwhile, si-circ_0011946\#1 and \#2 had hardly changed the level of linear SCMH1 (Fig. 2B). Si-circ_0011946\#1 with the most remarkable efficacy was chosen for succeeding investigations. Circ 0011946 knockdown markedly decreased cell proliferation by diminishing cell viability and colony formation capability (Fig. 2C and D). Besides, circ_0011946 silence evidently repressed cell migration and invasion (Fig. 2EG). Likewise, circ_0011946 depletion induced significant apoptosis (Fig. 2H). Hence, circ_0011946 knockdown repressed OSCC cell progression in vitro.

\section{Circ_0011946 targets miR-216a-5p in OSCC cells}

The target miRNAs of circ_0011946 were searched by starBase database. MiR-216a-5p was targeted by circ_ 


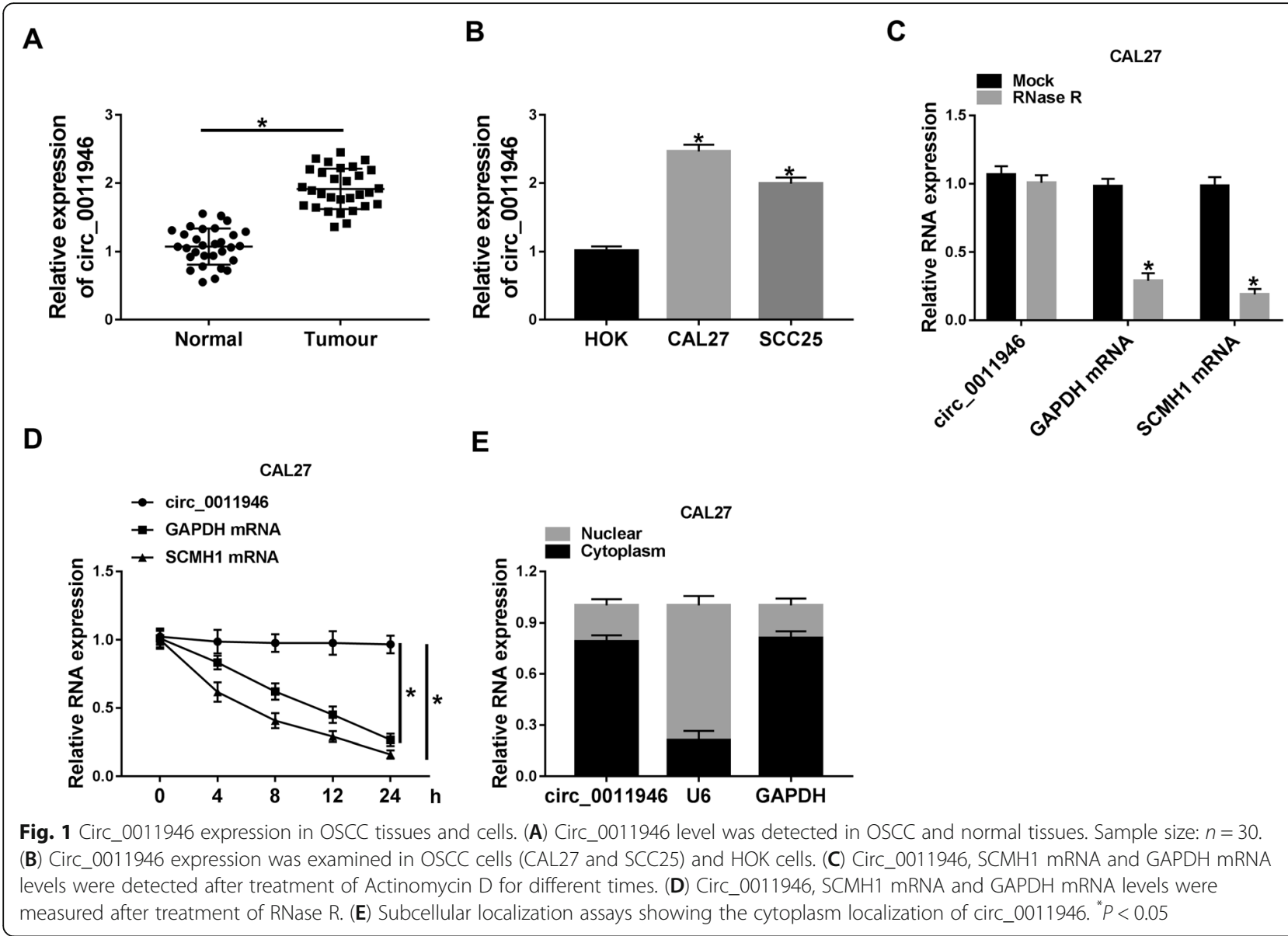

0011946, and the binding site of circ_0011946 and miR216a-5p was presented in Fig. 3A. MiR-216a-5p overexpression caused $\mathrm{a} \sim 70 \%$ diminution of luciferase activity in WT-circ_0011946 group, even though it makes no difference in the activity in MUT-circ_0011946 group (Fig. 3B). Likewise, RNA pull-down assay disclosed that circ_0011946 and miR-216a-5p were enriched by circ 0011946 probe (Fig. 3C and D). Additionally, miR-216a$5 \mathrm{p}$ abundance in OSCC was measured, and reduced miR-216a-5p level was disclosed in OSCC tissue samples and cell lines (Fig. 3E and F). The transfection efficacy of miR-216a-5p inhibitor was identified by qRT-PCR (Fig. 3G). Remarkably, miR-216a-5p level was elevated by circ_0011946 silence, whereas this impact was relieved by miR-216a-5p downregulation (Fig. 3H). Altogether, miR-216a-5p is the target of circ_0011946 in OSCC cells.

miR-216a-5p downregulation attenuates the influences of si-circ_0011946 on OSCC cell progression

To scrutinize if miR-216a-5p was accountable for the mediation of circ_0011946, CAL27 and SCC25 cells were introduced with si-NC, si-circ_0011946\#1, si-circ_ $0011946 \# 1+$ inhibitor NC or si-circ_0011946\#1 + miR-
$216 a-5 p$ inhibitor. Indeed, miR-216a-5p reduction mitigated si-circ_0011946-mediated suppression on cell proliferation (Fig. 4A and B). Furthermore, miR-216a-5p knockdown alleviated the inhibitory function of circ 0011946 downregulation on cell migration and invasion (Fig. 4C-E). Additionally, miR-216a-5p deletion diminished circ_0011946 silence-induced cell apoptosis (Fig. 4F). These data suggested that the effects of circ 0011946 silence were due to the up-regulation of miR216a-5p.

\section{Circ_0011946 regulates BCL2L2 expression by}

\section{competitively binding to miR-216a-5p}

The molecular targets of miR-216a-5p were forecasted by starBase database, and the data showed that BCL2L2 was a presumed target of miR-216a-5p (Fig. 5A). High level of miR-216a-5p diminished the luciferase activity of WT-BCL2L2-3'UTR by 75\%, whereas it exhibited little impact on MUT-BCL2L2-3'UTR group (Fig. 5B). Moreover, BCL2L2 mRNA and protein level were considerably heightened in OSCC tissues and cells (Fig. 5C and D). Additionally, the transfection efficacy of miR-216a$5 \mathrm{p}$ mimic was affirmed by qRT-PCR analysis (Fig. 5E). BCL2L2 expression was considerably diminished by 

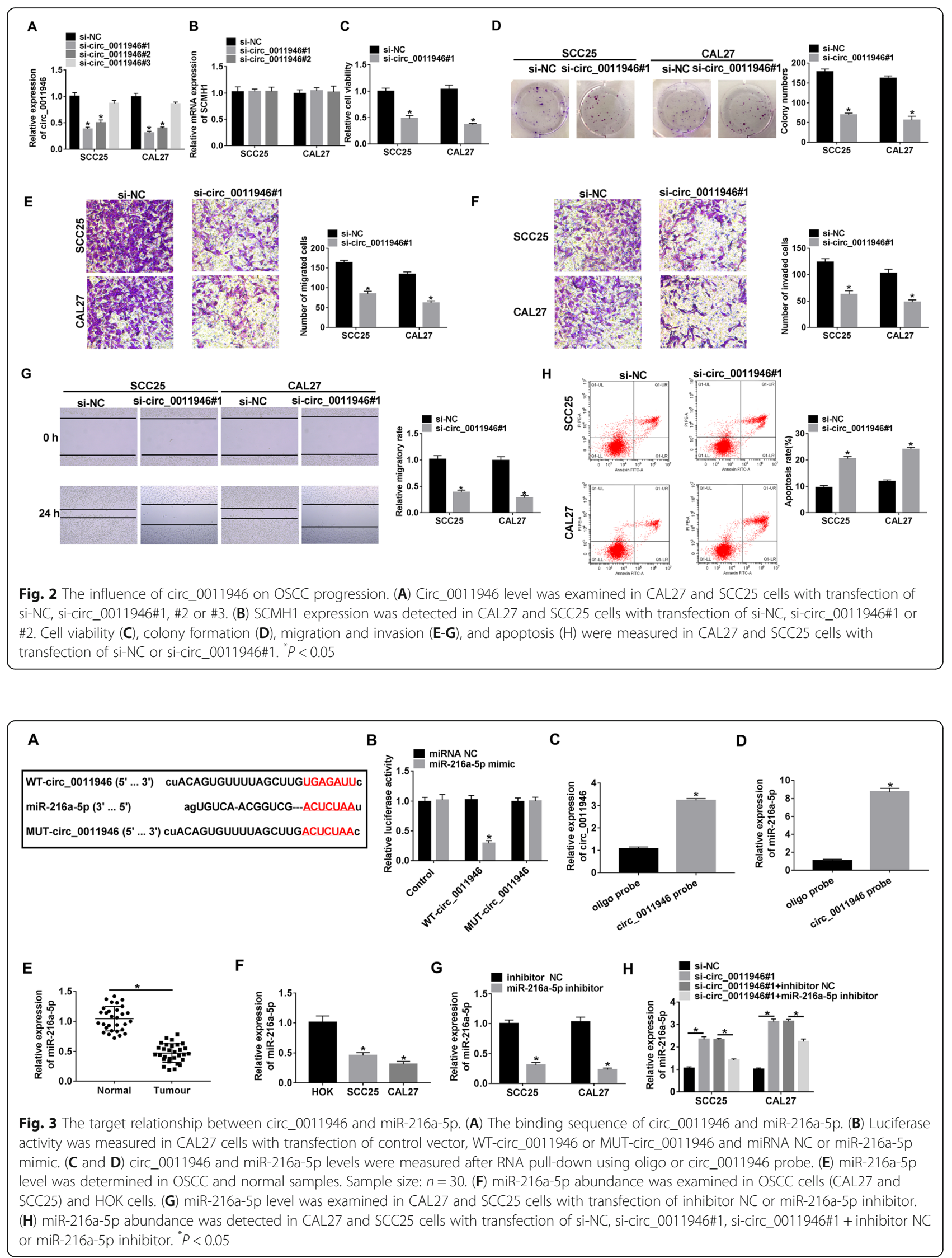

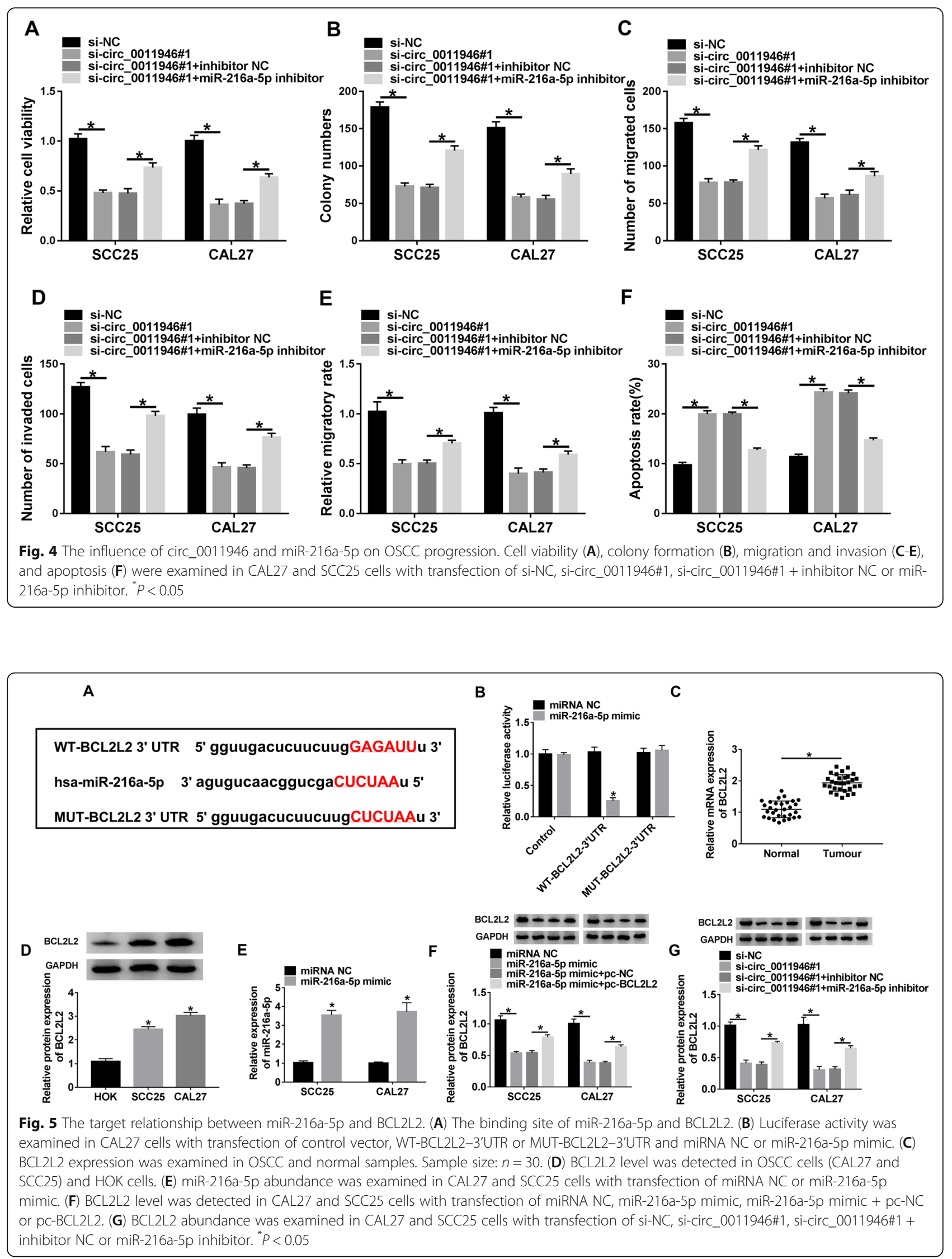
miR-216a-5p mimic introduction, which was repaired by transfection of pc-BCL2L2 (Fig. 5F). Additionally, the strong inverse correlation was found between BCL2L2 mRNA and miR-216a-5p expressions in OSCC tissues (Supplement Fig. 1). Furthermore, qRT-PCR results displayed that circ_0011946 silence resulted in decreased expression of BCL2L2 protein, and this influence was revoked by miR-216a-5p knockdown (Fig. 5G). Collectively, circ_0011946 mediated BCL2L2 expression by miR-216a-5p.

\section{MiR-216a-5p restrains OSCC cell development by managing $\mathrm{BCL} 2 \mathrm{~L} 2$}

To test the impacts of miR-216a-5p and BCL2L2 in the malignant phenotypes of OSCC, gain-of-function assays were fulfilled in CAL27 and SCC25 cells. Elevated expression of miR-216a-5p obviously constrained cell proliferation, which was reversed by BCL2L2 restoration (Fig. 6A and B). Additionally, miR-216a-5p overexpression impelled a notable inhibition on cell migratory and invasive abilities, and this effect was mitigated by BCL2L2 up-regulation (Fig. 6C-E). Moreover, miR-216a$5 \mathrm{p}$ overexpression notably fostered cell apoptosis, which was attenuated by introduction of BCL2L2 expressing plasmid (Fig. 6F). These data uncovered that miR-216a$5 p$ impacted OSCC cell behaviors by regulating BCL2L2.
Circ_0011946 knockdown reduces tumor growth in xenograft model

Subsequently, an in vivo xenograft model was utilized to examine the influence of circ_0011946 in OSCC cell growth. As displayed in Fig. 7A and B, tumor volume and weight of sh-circ_0011946 group were diminished than those in sh-NC group. Besides, circ_0011946 and BCL2L2 levels were lessened in sh-circ_0011946 group as opposed to sh-NC group, while miR-216a-5p level was increased (Fig. 7C-E). Additionally, sh-circ 0011946-transduced tumors had significantly fewer cells stained for Ki67 staining than the sh-NC control (Fig. 7E). These data showed that silencing of circ_0011946 minimized tumor growth in vivo.

\section{Discussion}

OSCC is the commonest type of oral cancers with high morbidity worldwide [18]. Many circRNAs have been revealed to apply important works in tumorigenesis and treatment of OSCC [6]. The objective of this study is to study new mechanism underlying the regulation of circ 0011946 in OSCC.

Meng et al. disclosed that circ_0011946 expression was strengthened in OSCC, and circ_0011946 silence restrained cell proliferation and metastasis in CAL27 cells [9]. Here, circ_0011946 abundance was elevated in OSCC. Moreover, circ_0011946 knockdown could

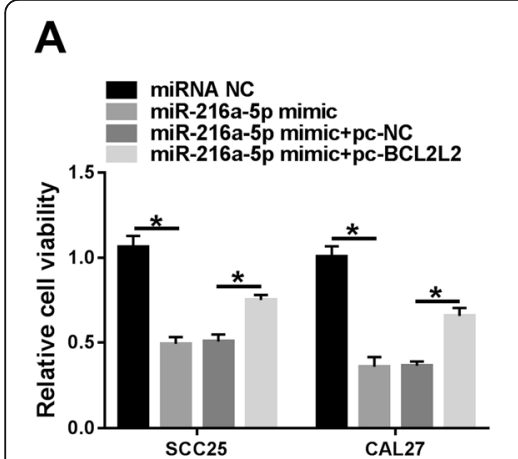

\section{B}

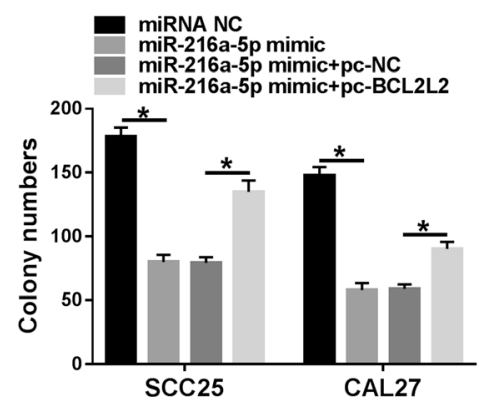

E
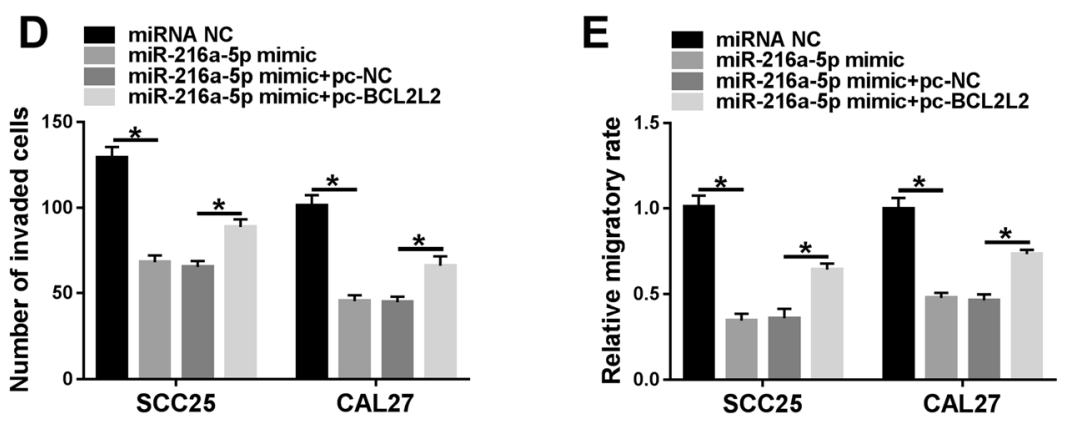

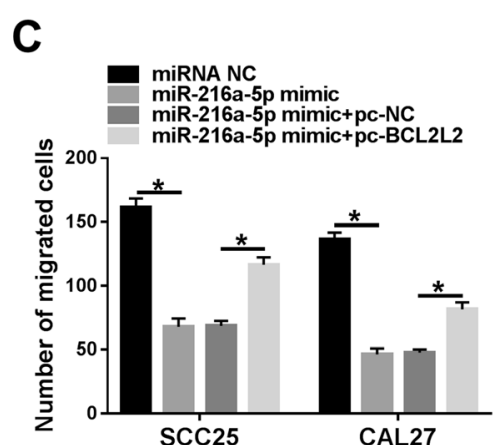

F

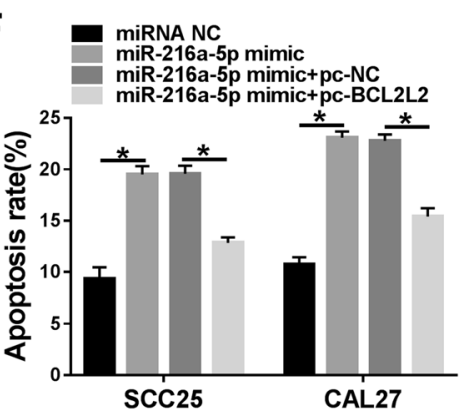

Fig. 6 The influence of miR-216a-5p and BCL2L2 on OSCC progression. Cell viability (A), colony formation (B), migration and invasion (C-E), and apoptosis (F) were examined in CAL27 and SCC25 cells with transfection of miRNA NC, miR-216a-5p mimic, miR-216a-5p mimic + pc-NC or pc-BCL2L2. ${ }^{*} P<0.05$ 


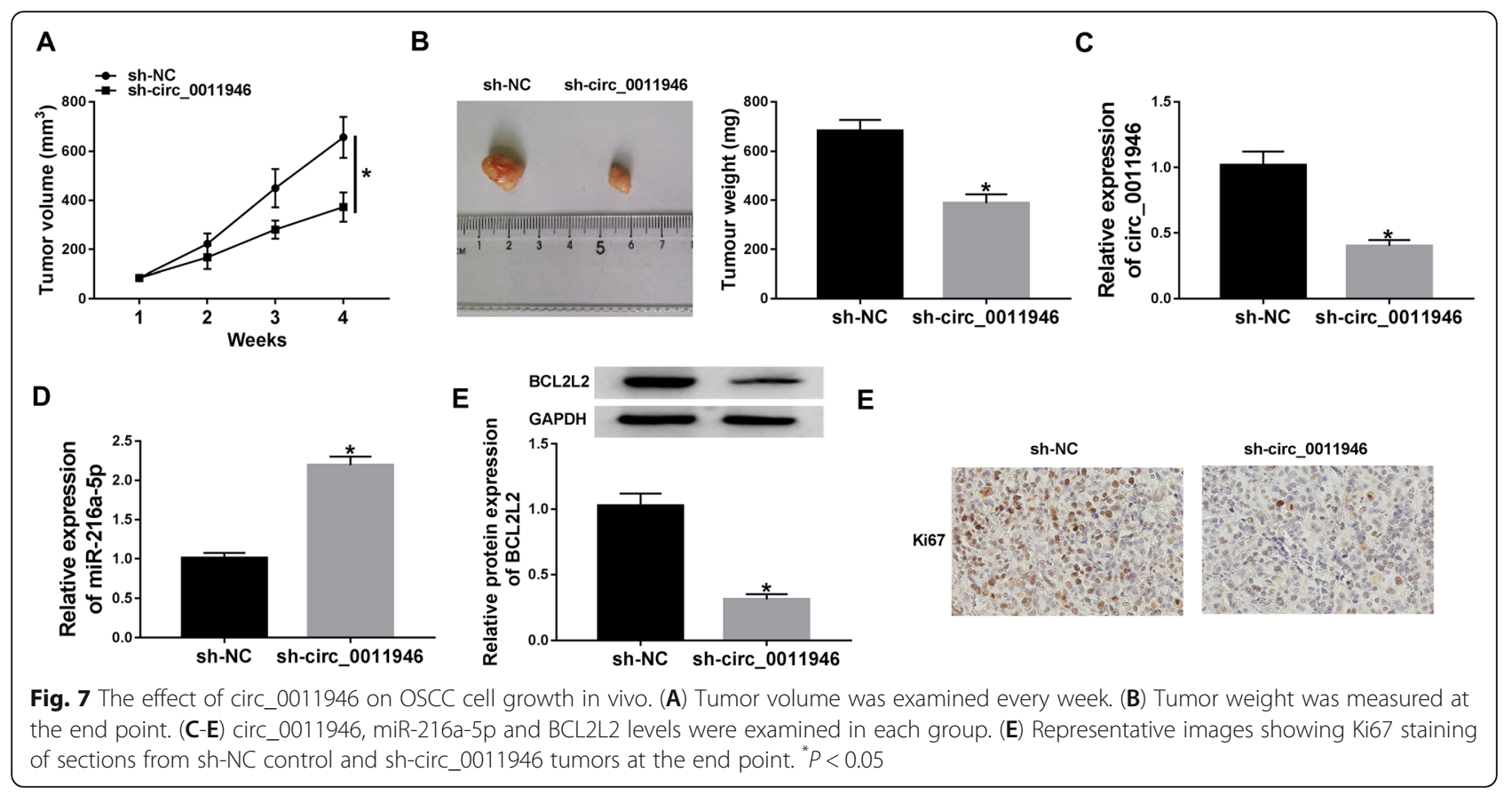

suppress cell proliferation, migration, and invasion, but promote apoptosis in CAL27 and SCC25 cells. This was also in agreement with that in other cancers, such as breast cancer and hepatocellular carcinoma [19, 20]. Additionally, in this study, we used siRNAs targeting circ_0011946 to silence circ_0011946, which are designed by targeting the sequence spanning the junction of circ_0011946. Since the sequence spanning the junction is unique for circ_0011946, the siRNAs do not affect the transcription of the linear mRNA.

Here, we ascertained that miR-216a-5p was sponged by circ_0011946. miR-216a-5p was disclosed to be lower expressed in OSCC, and restrained cell proliferation, migration and invasion [12]. Furthermore, overexpressed miR-216a-5p oppressed OSCC cell development, and its tumor suppressor function was also uncovered in breast cancer, esophageal squamous cell carcinoma, and osteosarcoma [21-24]. Additionally, our findings first demonstrated that the function of circ_0011946 depended, at least in part, on miR-216a-5p.

Next, we confirmed that BCL2L2 was a target and functional target of miR-216a-5p. BCL2L2 has established roles in promoting human tumorigenesis [14, 2527]. Previous work also reported the oncogenic role of BCL2L2 in OSCC [16]. Moreover, BCL2L2 has not only anti-apoptotic role but also promotes cell migration and invasion [13]. Multiple studies have reported that BCL2L2 enhanced cell migration and invasion in several forms of cancers [15, 28, 29]. Here, we first uncovered that circ_0011946 regulated BCL2L2 level by miR-216a$5 p$ competition. In addition, a xenograft model identified the anti-growth function of circ_0011946 silence in
OSCC. However, there was a limitation that we did not analyze the influence of miR-216a-5p/BCL2L2 axis on xenograft tumor growth in vivo, which will be studied in future work.

\section{Conclusions}

Taken together, our findings suggest that circ_0011946 knockdown constrains cell proliferation, invasion and migration, but promotes apoptosis in OSCC at least in part by the miR-216a-5p/BCL2L2 axis.

\section{Abbreviations}

circRNAs: circular RNAs; OSCC: oral squamous cell carcinoma; circ_0011946: circRNA hsa_circ_0011946; miR: microRNA; BCL2L2: B cell lymphoma-2-like 2 protein; mTOR: mammalian target of rapamycin; SCMH1: Scm polycomb group protein homolog 1; PCNA: proliferating cells nuclear antigen; miRNAs: microRNAs; EIF4B: eukaryotic translation initiation factor $4 \mathrm{~B}$

\section{Supplementary Information}

The online version contains supplementary material available at https://doi. org/10.1186/s12885-021-08779-4.

Additional file 1: Supplement Figure 1. Expression correlation between BCL2L2 mRNA and miR-216a-5p levels in OSCC tissues using the Pearson's rank correlation coefficient.

Acknowledgements

None.

Authors' contributions

YZ: Conceptualization and writing original draft. SZ and ZM: Formal analysis. $\mathrm{ZY}$ and HZ: data curation. YZ and JJ: Investigation and methodology. JJ: Supervision and validation. All authors have read and approved the manuscript. 
Funding

None.

\section{Availability of data and materials}

All data generated or analysed during this study are included in this published article [and its supplementary information files].

\section{Declarations}

\section{Ethics approval and consent to participate}

All participants signed the written informed consent. This research was authorized via the ethics committee of Jiaxing Hospital of Traditional Chinese Medicine, Jiaxing University.

\section{Consent for publication}

Not Applicable.

\section{Competing interests}

The authors declare that they have no competing interests.

\section{Author details}

'Department of Dentistry, Jiaxing Hospital of Traditional Chinese Medicine, Jiaxing University, No. 1501 Zhongshan East Road, Jiaxing, Zhejiang 314001, China. ${ }^{2}$ Department of Orthopaedics, Jiaxing Hospital of Traditional Chinese Medicine, Jiaxing University, No. 1501 Zhongshan East Road, Jiaxing, Zhejiang 314001, China.

\section{Received: 17 May 2021 Accepted: 13 September 2021}

Published online: 07 October 2021

\section{References}

1. Peres MA, Macpherson LMD, Weyant RJ, Daly B, Venturelli R, Mathur MR, et al. Oral diseases: a global public health challenge. Lancet. 2019; 394(10194):249-60. https://doi.org/10.1016/S0140-6736(19)31146-8.

2. Almangush A, Makitie AA, Triantafyllou A, de Bree R, Strojan P, Rinaldo A, et al. Staging and grading of oral squamous cell carcinoma: an update. Oral Oncol. 2020;107:104799. https://doi.org/10.1016/j.oraloncology.2020.104799.

3. Momen-Heravi F, Bala S. Emerging role of non-coding RNA in oral cancer. Cell Signal. 2018;42:134-43. https://doi.org/10.1016/j.cellsig.2017.10.009.

4. Kristensen LS, Andersen MS, Stagsted LWW, Ebbesen KK, Hansen TB, Kjems J. The biogenesis, biology and characterization of circular RNAs. Nat Rev Genet. 2019;20(11):675-91. https://doi.org/10.1038/s41576-019-0158-7.

5. Li J, Sun D, Pu W, Wang J, Peng Y. Circular RNAs in Cancer: biogenesis, function, and clinical significance. Trends Cancer. 2020;6(4):319-36. https:// doi.org/10.1016/j.trecan.2020.01.012.

6. Cristobal I, Carames C, Rubio J, Sanz-Alvarez M, Luque M, Madoz-Gurpide J, Rojo F, Garcia-Foncillas J: Functional and Clinical Impact of CircRNAs in Oral Cancer. Cancers (Basel) 2020, 12(4), 12, 4, https://doi.org/10.3390/cancers12 041041.

7. Peng QS, Cheng YN, Zhang WB, Fan H, Mao QH, Xu P. circRNA_0000140 suppresses oral squamous cell carcinoma growth and metastasis by targeting miR-31 to inhibit hippo signaling pathway. Cell Death Dis. 2020; 11(2):112. https://doi.org/10.1038/s41419-020-2273-y.

8. Yang Y, Ci HS, Mao YL, Li JW, Zuo JH. CircRNA_002178 promotes the proliferation and migration of oral squamous cell carcinoma cells by activating the Akt/mTOR pathway. Eur Rev Med Pharmacol Sci. 2020;24(11): 6122-30. https://doi.org/10.26355/eurrev_202006_21507.

9. Meng Y, Zhao EY, Zhou Y, Qiang DX, Wang S, Shi L, et al. Circular RNA hsa_ circ_0011946 promotes cell growth, migration, and invasion of oral squamous cell carcinoma by upregulating PCNA. Eur Rev Med Pharmacol Sci. 2020;24(3):1226-32. https://doi.org/10.26355/eurrev_202002_20175.

10. Min A, Zhu C, Peng S, Rajthala S, Costea DE, Sapkota D. MicroRNAs as important players and biomarkers in Oral carcinogenesis. Biomed Res Int. 2015;2015:186904-10. https://doi.org/10.1155/2015/186904.

11. Ghosh RD, Pattatheyil A, Roychoudhury S. Functional landscape of dysregulated MicroRNAs in Oral squamous cell carcinoma: clinical implications. Front Oncol. 2020;10:619. https://doi.org/10.3389/fonc.2020, 00619.

12. Li L, Ma HQ. MicroRNA-216a inhibits the growth and metastasis of oral squamous cell carcinoma by targeting eukaryotic translation initiation factor
4B. Mol Med Rep. 2015;12(2):3156-62. https://doi.org/10.3892/mmr.2015.3 761.

13. Hartman ML, Czyz M. BCL-w: apoptotic and non-apoptotic role in health and disease. Cell Death Dis. 2020;11(4):260. https://doi.org/10.1038/s41419020-2417-0.

14. Ming $M$, Ying $M$. Ling M: miRNA-125a-5p inhibits hepatocellular carcinoma cell proliferation and induces apoptosis by targeting TP53 regulated inhibitor of apoptosis 1 and Bcl-2-like-2 protein. Exp Ther Med. 2019;18(2): 1196-202. https://doi.org/10.3892/etm.2019.7674.

15. Yang T, Thakur A, Chen T, Yang L, Lei G, Liang Y, et al. MicroRNA-15a induces cell apoptosis and inhibits metastasis by targeting BCL2L2 in nonsmall cell lung cancer. Tumour Biol. 2015;36(6):4357-65. https://doi.org/10.1 007/s13277-015-3075-1.

16. Wang X, Li GH. MicroRNA-16 functions as a tumor-suppressor gene in oral squamous cell carcinoma by targeting AKT3 and BCL2L2. J Cell Physiol. 2018;233(12):9447-57. https://doi.org/10.1002/jcp.26833.

17. Livak KJ, Schmittgen TD. Analysis of relative gene expression data using real-time quantitative PCR and the 2(-Delta Delta C(T)) method. Methods. 2001;25(4):402-8. https://doi.org/10.1006/meth.2001.1262.

18. Rivera C, Oliveira AK, Costa RAP, De Rossi T, Paes Leme AF. Prognostic biomarkers in oral squamous cell carcinoma: a systematic review. Oral Oncol. 2017;72:38-47. https://doi.org/10.1016/j.oraloncology.2017.07.003.

19. Zhou J, Zhang WW, Peng F, Sun JY, He ZY, Wu SG. Downregulation of hsa_ circ_0011946 suppresses the migration and invasion of the breast cancer cell line MCF-7 by targeting RFC3. Cancer Manag Res. 2018;10:535-44. https://doi.org/10.2147/CMAR.S155923.

20. Ren L, Zhai H, Wang XL, Li JZ, Xia YH. Hsa_circ_0011946 promotes the migration and invasion of hepatocellular carcinoma by inducing EMT process. Eur Rev Med Pharmacol Sci. 2020;24(3):1108-15. https://doi.org/10.2 6355/eurrev_202002_20161.

21. Liu W, Huang L, Zhang C, Liu Z. IncRNA MEG3 is downregulated in ankylosing spondylitis and associated with disease activity, hospitalization time and disease duration. Experimental and therapeutic medicine. 2019; 17(1):291-7. https://doi.org/10.3892/etm.2018.6921.

22. Chai L, Yang G. MiR-216a-5p targets TCTN1 to inhibit cell proliferation and induce apoptosis in esophageal squamous cell carcinoma. Cell Mol Biol Lett. 2019;24(1):46. https://doi.org/10.1186/s11658-019-0166-9.

23. Zeng X, Liu Y, Zhu H, Chen D, Hu W. Downregulation of miR-216a-5p by long noncoding RNA PVT1 suppresses colorectal cancer progression via modulation of YBX1 expression. Cancer Manag Res. 2019;11:6981-93. https://doi.org/10.2147/CMAR.S208983.

24. Zhang X, Ji S, Cai G, Pan Z, Han R, Yuan Y, et al. H19 increases IL-17A/IL-23 releases via regulating VDR by interacting with miR675-5p/miR22-5p in ankylosing spondylitis. Mole Ther Nucl Acids. 2020;19:393-404. https://doi. org/10.1016/j.omtn.2019.11.025

25. Lu W, Feng L, Zhang Y, Ma Y, Li P, Wang Y, et al. Zhao G et al: miR-15a induces cell apoptosis by targeting BCL2L2 and BCL2 in HPV-positive hypopharyngeal squamous cell carcinoma. Oncol Rep. 2016;36(4):2169-76. https://doi.org/10.3892/or.2016.5049.

26. Wang C, Zhou B, Liu M, Liu Y. Gao R: miR-126-5p restoration promotes cell apoptosis in cervical Cancer by targeting Bcl212. Oncol Res. 2017;25(4):46370. https://doi.org/10.3727/096504016X14685034103879.

27. Zhang H, He QY, Wang GC, Tong DK, Wang RK, Ding WB, et al. miR-422a inhibits osteosarcoma proliferation by targeting BCL2L2 and KRAS. Biosci Rep. 2018;38(2):38(2). https://doi.org/10.1042/BSR20170339.

28. Cao J, Cai J, Huang D, Han Q, Yang Q, Li T, et al. Wang Z: miR-335 represents an invasion suppressor gene in ovarian cancer by targeting $\mathrm{BCl}-$ w. Oncol Rep. 2013;30(2):701-6. https://doi.org/10.3892/or.2013.2482.

29. Chung HJ, Choi YE, Kim ES, Han YH, Park MJ. Bae IH: miR-29b attenuates tumorigenicity and stemness maintenance in human glioblastoma multiforme by directly targeting BCL2L2. Oncotarget. 2015;6(21):18429-44. https://doi.org/10.18632/oncotarget.4384.

\section{Publisher's Note}

Springer Nature remains neutral with regard to jurisdictional claims in published maps and institutional affiliations. 